\title{
Force-Velocity Profiles of Elite Athletes Tested on a Cycle Ergometer
}

\author{
Predrag R. Bozic ${ }^{1,2}$, Bobana Berjan Bacvarevic ${ }^{1}$
}

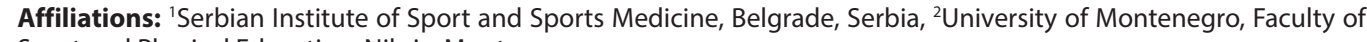
Sport and Physical Education, Niksic, Montenegro

Correspondence: P.R. Bozic, Serbian Institute of Sport and Sports Medicine, 72 Kneza Viseslava Street, 11030 Belgrade, Serbia.E-mail: predrag.bozic@rzsport.gov.rs

ABSTRACT The present study explored the sensitivity of the force-velocity (F-V) modelling approach obtained from maximal sprints on a leg cycle ergometer to detect selective changes of the mechanical capacities of the lower body muscles associated with high-level training. Specifically, we assumed that the F-V relationship parameters, such as maximum force $\left(\mathrm{F}_{0}\right)$, velocity $\left(\mathrm{V}_{0}\right)$, power $\left(\mathrm{P}_{\mathrm{M}}\right)$ and slope, would differ among individuals of different high-level training backgrounds. In total, 111 elite athletes divided into four groups (Combat sports, Athletic sprints, Team sports and Physically active) performed maximal sprints on a leg cycle ergometer loaded with $7 \%, 9 \%$, and $11 \%$ of body weight. The findings obtained suggest an exceptionably strong and linear F-V relationship in most of the participants $(r>0.95)$, while higher $\mathrm{P}_{\mathrm{M}}$ have been found in all groups of athletes compared to the Physically active group $(\mathrm{p}<0.05)$. In addition, sport-specific F-V profiles have been observed in athletes that belong to distinctively different sports (i.e. higher $\mathrm{F}_{0}$ and forceoriented slope for strength-trained Combat sports and higher $\mathrm{V}_{0}$ for speed-trained Athletic sprints). To our knowledge, this is one of the rare studies that evaluate the F-V profiles with such a large sample of elite athletes obtained from commonly used task such as maximal sprints on a leg cycle ergometer. The results obtained support a high sensitivity of the F-V modelling approach to distinguish among elite athletes with different training histories.

KEY WORDS sprint cycling test, force-velocity relationship, sensitivity, linear regression, elite athletes

@MJSSMontenegro

FORCE-VELOCITY PROFILES OF ELITE ATHLETES

http://mjssm.me/?sekcija=article\&artid=153

\section{Introduction}

For the successful performance of various functional movement tasks from daily life or sport, muscle mechanical capacities to produce high levels of force, velocity, or power are essential. However, the testing of muscle capacities has been routinely performed under a single pre-defined movement condition that allows obtaining single outcomes, such as the jump height, exerted force, or cycling frequency. As a result, selective assessment of the partly independent muscle capacities for producing maximal force $\left(\mathrm{F}_{0}\right)$, velocity $\left(\mathrm{V}_{0}\right)$ or power $\left(\mathrm{P}_{\mathrm{M}}\right)$ could not be possible through a single outcome (Jaric, 2015), since it changes substantially with load and movement velocity due in part to F-V and P-V relations (Westing, Seger, \& Thorstensson, 1990). This certainly leads to a fundamental problem in the contemporary literature and practice regarding both the testing procedures and the interpretation of results. To address the discussed problem, researchers occasionally apply test batteries that include different tasks such as sprints, repetition maximum tests, vertical jump tests, etc., or perform a specific task under different loading conditions. The assumption that greater performance in different tests or a specific task performed with heavy and light loads would be indicative of the better $\mathrm{F}_{0}$ and $\mathrm{V}_{0}$, respectively, could not be properly supported by the quantitative outcomes provided by independent tests or loading conditions.

A more promising solution of the discussed problem could be based on recent research focused upon the modelling of the F-V relationship of muscular system with performing different functional tasks or sport activities under two or more loading conditions. Specifically, the loaded functional multi-joint movements (e.g. 
jumping, walking, running, cycling, lifting, throwing) typically display a strong and linear F-V relationship of the tested muscles (Jaric, 2015; Vandewalle, Péerès, \& Monod, 1987). This enables determining the distinctive muscle capacities to produce $\mathrm{F}_{0}, \mathrm{~V}_{0}$, their ratio (the relationship slope), and $\mathrm{P}_{\mathrm{M}}$ (Jaric, 2015). The parameters obtained from the linear F-V relationship have consistently revealed high reliability (Jaric, 2015). In addition, the abilities to generate high movement velocity or resistance to high external load have been shown to be determined by $\mathrm{V}_{0}$ (Feeney, Stanhope, Kaminski, Machi, \& Jaric, 2016) and $\mathrm{F}_{0}$ (Driss, Vandewalle, Le Chevalier, \& Monod, 2002), respectively, while ballistic performance is largely dependent not only on $\mathrm{P}_{\mathrm{M}}$ (Samozino, Rejc, Di Prampero, Belli, \& Morin, 2012; Vandewalle, Peres, Heller, Panel, \& Monod, 1987) but also on an optimum balance between $\mathrm{F}_{0}$ and $\mathrm{V}_{0}$ (i.e., F-V slope) (Jiménez-Reyes, Samozino, Brughelli, \& Morin, 2017; Samozino et al., 2014).

These findings suggest that chronic exposure to training programs based on high movement velocity, explosive movements, or resistance exercise would result in selective changes in $\mathrm{F}_{0}, \mathrm{~V}_{0}, \mathrm{P}_{\mathrm{M}}$, and slope. Although there are many studies in which various maximum performance functional tasks have been used to assess the different training effects or distinguish among various populations (for review, see Cormie, McGuigan, \& Newton, 2011a, 2011b), there is a lack of research that has examined sensitivity of the F-V relationship parameters to detect different training adaptations (Jaric, 2016). To the best of our knowledge, the only several studies have examined sensitivity of the F-V relationship parameters to distinguish among individuals with different training backgrounds (Cuk et al., 2016; Giroux, Rabita, Chollet, \& Guilhem, 2016; Ravier, Grappe, \& Rouillon, 2004; Vandewalle et al., 1987). Evaluating the F-V relationship through maximal sprints on the leg cycle ergometer, Vandewalle et al. (1987) revealed a greater $\mathrm{P}_{\mathrm{M}}$ and $\mathrm{V}_{0}$ for fast and powerful athletes in comparison to endurance athletes. In addition, better $\mathrm{P}_{\mathrm{M}}$ and $\mathrm{V}_{0}$ capacities have been observed in karate athletes at higher competition levels (Ravier et al., 2004). Using vertical jumps for the evaluation of the F-V relationship, Cuk et al. (2016) found higher $\mathrm{P}_{\mathrm{M}}$ and $\mathrm{F}_{0}$ capacities in strength-trained athletes in comparison to physically active and sedentary persons. Evaluating various elite athletes, Giroux et al. (2016) also found that the chronic practice of specific sports leads to differently balanced force-velocity profiles. Although the mentioned findings support the existence of the selective changes in the F-V profile regarding a specific focus of long-term training, for a better understanding of the mentioned problem, further research is required. It seems of interest to elucidate subtle changes in the F-V relationship of various elite practitioners whose training in high-level sport has been focused mainly on improving the power production capacity: the power output can be increased either by improving the capacity to develop a higher level of force in the same period (i.e. improving $\mathrm{F}_{0}$ ) or to develop the same amount of force over a shorter duration (i.e. improving $\left.\mathrm{V}_{0}\right)(\mathrm{Newton}$ \& Kraemer, 1994). Therefore, the F-V modelling approach could be appropriate to reveal specific training adaptation through any changes in $\mathrm{F}_{0}, \mathrm{~V}_{0}, \mathrm{P}_{\mathrm{M}}$ and slope. Particularly, it would be important to assess the sensitivity of the F-V modelling approach obtained from commonly used testing modality such as maximal sprints performed on a leg cycle ergometer. When compared with other functional tasks used to evaluate the F-V relationship of leg extensors (e.g. vertical jumps, running, different types of closed kinetic chain leg extension movements, etc.; Jaric, 2015), the maximal sprints performed on a cycle friction ergometer allows a simple and accurate manipulation of external loading and, therefore, it is used as the preferred task for the evaluation of muscle mechanical capacities in numerous studies (Pazin, Bozic, Bobana, Nedeljkovic, \& Jaric, 2011; Henry Vandewalle et al., 1987).

To address the problems discussed above, we explored the sensitivity of the F-V relationship obtained from maximal sprints on a leg cycle ergometer to detect selective changes on the mechanical capacities of the lower body muscles associated with high-level training. We hypothesized that (H1) sports in which muscle capacities greatly determine success would reveal higher $\mathrm{P}_{\mathrm{M}}$ in comparison to other sports. Regarding the changes in the F-V relationship shift associated with chronic practice in a specific sport, we hypothesized that (H2) the sports mainly focused on developing power production at lower relative loads and higher velocities would reveal velocity-oriented profile, while sports focused on developing power at heavier relative loads would reveal a force-oriented profile.

\section{Methods}

Subjects

One hundred and eleven elite athletes were selected to participate in this study. They were allocated into four groups according to their training history: Combat sports (Chaabene et al., 2016; including wrestling and judo athletes; mainly focused on developing power production at heavier relative loads; Franchini, Del Vecchio, Matsushigue, \& Artioli, 2011; Tabben et al., 2014), Athletic sprints (including athletes form track and field sprint disciplines 60-400 m; mainly focused on developing power production at lower relative loads; Morin et al., 2012), Team sports (including athletes from team sports, such as volleyball, handball, and basketball; high levels of muscle capacities are desirable, but not always necessary since technical and tactical skills greatly determine performance; Apostolidis, Nassis, Bolatoglou, \& Geladas, 2004; Gabbett, Georgieff, \& Domrow, 2007; Massuça \& Fragoso, 2013) and Physically active (including athletes from auto racing and shooting; without significant requirements for production of maximum force, velocity, or power). The subjects' characteristics are depicted in Table 1. The sample of participants consisted of members of national selections, medallists in either international or national championships. None of the participants reported any medical problems or recent injuries that could compromise the tested performance. The participants 
were informed regarding the potential risks associated with the applied testing protocol and asked to sign an informed consent document prior to the testing protocol. The study was approved by the Institutional Review Board of the Serbian Institute of Sport and Sports Medicine, and carried out according to the Declaration of Helsinki.

\section{Testing procedures}

Anthropometric measures were taken according to the procedures recommended by the International Society for the Advancement of Kinanthropometry (Norton et al., 2000). Body height and body mass were measured to the nearest $0.5 \mathrm{~cm}$ and $0.1 \mathrm{~kg}$, respectively. Testing of the force-velocity relationship was performed through the 6-s maximal cycling sprint test (Logan, Fornasiero, Abernethy, \& Lynch, 2000; Mendez-Villanueva, Bishop, \& Hamer, 2007) on a Monark 834E leg cycle ergometer (Monark, Varberg, Sweden). Several studies confirmed high test-retest reliability of this $(r=0.98$; Wilson, Newton, Murphy, \& Humphries, 1993) and similar sprint cycling tests (Dotan \& Bar-Or, 1983; r = 0.89-0.96; Evans \& Quinney, 1981; Patton, Murphy, \& Frederick, 1985), while Mendez-Villanueva et al. (2007) found low within-subject variations when the 6-s maximal cycling sprint test was preceded by a familiarization session $(\mathrm{CV}<2 \%)$. One potential advantage of the selected cycling test could also be that it was non-specific for each group of participants. Finally, when compared with other standard tests, such as Margaria, vertical jumps, isokinetic testing, the selected 6-s maximal cycling sprint test allows a simple and accurate manipulation of external loading. The maximal 6-s cycling sprints were performed with three different loads: 7, 9, and $11 \%$ of body weight (BW). Prior to the test, subjects performed a standardized warm-up procedure comprising 5-min of cycling. A self-selected cadence against $2 \%$ of BW frictional load was applied to the flywheel, followed by 3 -min of easy stretching of the musculature of the lower extremities. Finally, a specific warm-up protocol consisting of two bouts of 3-s maximal acceleration separated by 3 -min rest were applied. Following a 5 -min recovery period, the subjects performed three 6-s sprints against different loads in a random sequence. They were instructed to perform an "all out" effort from the very beginning of the test until instructed to stop. The seat height was adjusted to each participant's satisfaction, and toe clips with straps were used to prevent the feet from slipping off the pedals. The start position on the cycle ergometer was strictly standardized: the subject was seated on the saddle during the sprint and initiated the exercise with his preferred leg, the crank was located at $45^{\circ}$ forward. Strong verbal encouragement was provided during each trial. The rest period among consecutive sprints was 4-min. Fatigue was never an issue.

The subjects were asked to follow their normal diet and to refrain from any form of intense physical activity for $48 \mathrm{~h}$, as well as to fast for $2 \mathrm{~h}$ prior to each testing session.

\section{Data analyses}

The device software was used to acquire power and pedalling frequency presented as a revolution per minute (rpm). The power and frequency data were calculated for every revolution of the flywheel and were continuously presented as a mean value of one second. To assess the corresponding F-V relationship, we previously perform several calculations to obtain $\mathrm{V}$ and $\mathrm{F}$ data. $\mathrm{V}$ was calculated from pedalling frequency $(\mathrm{rpm})$ and the crank length $(\mathrm{r}=0.17 \mathrm{~m})$ :

$$
V=\frac{r m p}{60 \times 2 r \pi}
$$

F was calculated as $\mathrm{P}$ divided by V. For further calculations, we used values of $\mathrm{V}$ and $\mathrm{F}$ at the instant of maximal pedalling frequency during $6 \mathrm{~s}$. To avoid the effect of body size dimensions on test results, indices of force were normalized for body size using the body mass raised to the power of 0.67 (i.e. in N/kg0.67; Jaric, 2002) providing the normalized values of $F$.

Linear regression methods were used for modelling of the F-V relationship. Therefore, using $\mathrm{X}=$ velocity $(\mathrm{m} / \mathrm{s}), \mathrm{Y}=$ force $(\mathrm{N})$, and $\mathrm{b} 0$ and $\mathrm{b} 1=$ statistically determined regression coefficients, the regression is:

$$
Y=b_{0}+b_{1} X
$$

The F-V relationships were extrapolated to determine the maximum force ( $\mathrm{F}_{0}$; force or Y-intercept), maximum velocity $\left(\mathrm{V}_{0}\right.$; velocity or $\mathrm{X}$-intercept), maximum power

$$
P_{M}=\frac{F_{0} \times V_{0}}{4}
$$

as well as the slope of the relationship

$$
a=\frac{F_{0}}{V_{0}}
$$




\section{Statistical analyses}

Standard descriptive statistics were calculated for all depended variables and groups. The Levene test was used to verify the homogeneity of variance for each variable analysed. One-way analysis of variance (ANOVA) with Hochberg's GT2 post hoc comparisons test was applied to assess between-group differences of the regression parameters $\left(\mathrm{F}_{0}, \mathrm{~V}_{0}, \mathrm{P}_{\mathrm{M}}\right.$ and slope). The effect size was used to estimate the magnitude of differences of the main

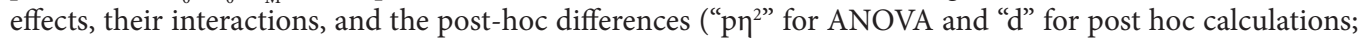
Cohen, 1988). The differences were considered as either small $\left(\mathrm{p}^{2}=0.01 ; \mathrm{d}=0.2\right)$, moderate $\left(\mathrm{p} \eta^{2}=0.06\right.$; $\mathrm{d}=0.5)$, or large $\left(\mathrm{p \eta}^{2}=0.15 ; \mathrm{d}=0.8\right)$. In addition, statistical power of difference (i.e. $\left.1-\beta\right)$ was calculated. Statistical significance was set at $\mathrm{p}<0.05$ and the data were analysed using SPSS (v. 21.0, IBM, Armonk, New York, USA).

\section{Results}

Figure 1 shows the typical F-V and P-V relationships obtained from representative individuals of the four different sport groups during maximum pedalling activity performed at different applied loads. As expected, the force data declined with increasing movement velocity. The force-velocity relationship has been found to be an exceptionably strong and approximately linear in most of the participants $(r>0.95)$ while the corresponding power-velocity relationship could be optimally explained by the second-order polynomial regression.

\begin{tabular}{lllll}
\multicolumn{7}{l}{ TABLE 1. Subjects' } & \multicolumn{4}{l}{ characteristics $($ mean \pm SD) } \\
Variable & $\begin{array}{l}\text { Combat sports } \\
(\mathbf{n = 2 0 )}\end{array}$ & $\begin{array}{l}\text { Athletic sprints } \\
(\mathbf{n = 9 )}\end{array}$ & $\begin{array}{l}\text { Team sports } \\
(\mathbf{n}=\mathbf{3 9 )}\end{array}$ & $\begin{array}{l}\text { Physically active } \\
(\mathbf{n}=\mathbf{4 3})\end{array}$ \\
\hline Height $(\mathrm{cm})$ & $177.9 \pm 7.1$ & $185.1 \pm 4.8$ & $193.5 \pm 9.1$ & $180.4 \pm 7.6$ \\
Body mass $(\mathrm{kg})$ & $78.8 \pm 8.7$ & $77.2 \pm 9.1$ & $86.5 \pm 11.4$ & $77.8 \pm 12.1$ \\
Age (years) & $22.2 \pm 2.9$ & $20.4 \pm 3.5$ & $20.9 \pm 3.5$ & $22.4 \pm 4.3$ \\
\hline
\end{tabular}

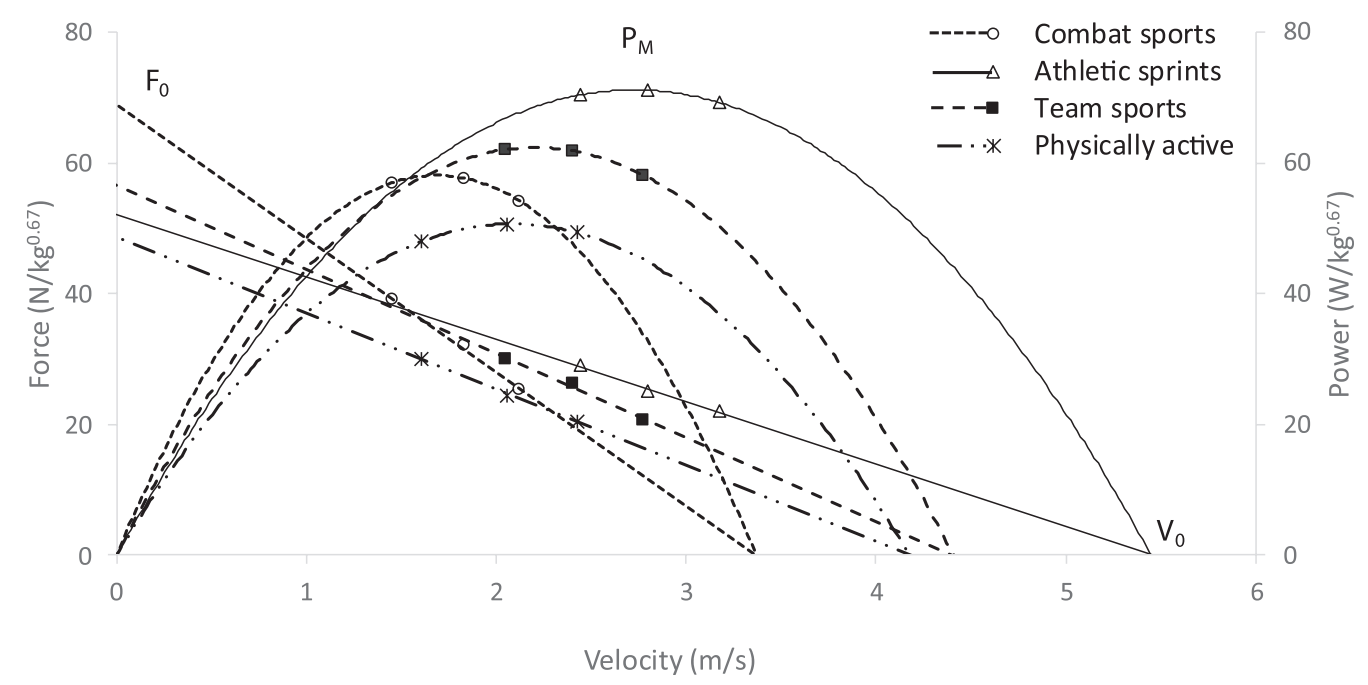

FIGURE 1 Typical example of the linear force-velocity and the polynomial power-velocity relationships, obtained during maximal sprinting on a leg friction-loaded cycle ergometer from 3 loading conditions (7\%, 9\%, and 11\% of body weight) for representative individuals of the four groups.

Figure 2 illustrates the main findings of the present study. The sensitivity analysis was based on the data set obtained from four groups $(\mathrm{N}=111)$ distinguished either by their training history or by the level of physical activity. The residuals of dependent variables observed in the F-V test appeared to be normally distributed ( $\mathrm{p}$ $>0.40$ ). The values for different groups (Combat sports vs Athletic sprints vs Team sports vs Physically active) are shown separately for slope (panel a), $\mathrm{F}_{0}$ (panel b), $\mathrm{V}_{0}$ (panel c), and $\mathrm{P}_{\mathrm{M}}$ (panel d). Significant differences among groups were obtained for the all evaluated variables, slope $\left(\mathrm{F}_{3,110}=3.37 ; \mathrm{p}=0.02 ; \mathrm{p} \eta^{2}=0.09 ; 1-\beta=\right.$ $0.75), \mathrm{F}_{0}\left(\mathrm{~F}_{3,110}=4.89 ; \mathrm{p}=0.00 ; \mathrm{p}^{2}=0.12 ; 1-\beta=0.90\right) \mathrm{V}_{0}\left(\mathrm{~F}_{3,110}=5.10 ; \mathrm{p}=0.00 ; \eta^{2}=0.13 ; 1-\beta=0.91\right)$ and $\mathrm{P}_{\mathrm{M}}\left(\mathrm{F}_{3,110}=10.78 ; \mathrm{p}=0.00 ; \mathrm{p}^{2}=0.23 ; 1-\beta=1.00\right)$. The post-hoc analysis suggested that the Combat sports group revealed force-oriented slope and higher values of $\mathrm{F}_{0}$ than Physically active did, while the Athletic sprints showed the highest values of $\mathrm{V}_{0}$ in comparison to all groups. Physically active were less powerful in comparison to other groups while moderate differences in $\mathrm{P}_{\mathrm{M}}$ were obtained between groups of Athletic sprints and Team sports. 

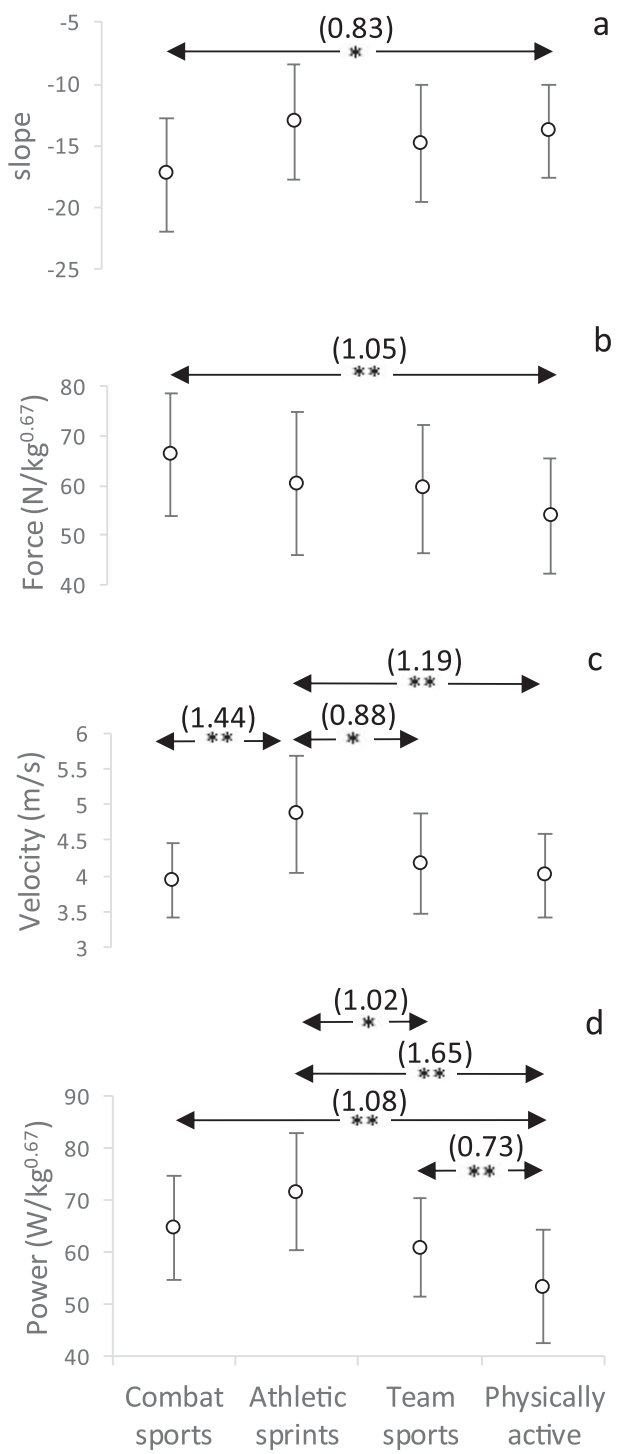

a

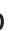

C
FIGURE 2 Averaged values of the regression parameters, slope (panel a), FMAX (panel b), VMAX (panel c) and PMAX (panel d), for different groups of subjects (mean with $\mathrm{SD}$ error bars). The lines with arrows depict differences between groups at $p<0.05\left(^{*}\right)$ and $p<0.01\left(^{* *}\right)$ with corresponding effect sizes in parentheses.

\section{Discussion}

To our knowledge, this is one of the rare studies that evaluate the F-V profiles in such a large and differentiated sample of elite athletes obtained from commonly used tasks, such as maximal sprints on a leg cycle ergometer. Specifically, we tested the hypothesized differences among elite athletes with distinct training histories. Overall, the obtained findings suggest an exceptionably strong and approximately linear force-velocity relationship in most of the participants, while both significant differences and mainly large effect sizes in power production capacities have been observed not only between groups of athletes and physically active participants, but also between groups of athletic sprinters and team sport athletes. These findings approved the first hypothesis of the present study. In addition, sport-specific F-V profiles have been observed for athletes that belong to distinctively different sports (i.e. force-oriented profile for strength-trained combat athletes and velocityoriented profile for speed-trained athletic sprinters) that proved the second hypothesis. Both the individual findings and their implications will be discussed in the following text.

In support of the first hypothesis, elite athletes participated in sports in which muscle capacities determine success (i.e. Combat sports, Athletic sprints and Team sports) produced higher $\mathrm{P}_{\mathrm{M}}$ than control group of athletes that are not characterized with outstanding muscle capacities (in this study labelled as Physically active). The obtained findings are in line with similar studies that show significant differences in $\mathrm{P}_{\mathrm{M}}$ between strength, speed or power-trained athletes compared to physically active or sedentary individuals (Cuk et al., 2016; Giroux et al., 2016; Pazin et al., 2011). In addition, the higher power-producing capacities of Athletic sprints group in comparison to Team sports group could also be expected since team sports allow slightly reduced muscle capacities compensated by outstanding technical and tactical skills (Reilly, Williams, Nevill, \& Franks, 2000). Regarding the second hypothesis, the force-oriented slope and consequently higher $\mathrm{F}_{0}$ was observed in the Combat sports group compared to the Physically active group. Traditionally, grappling combat sports, such as judo and wrestling, rely more on a high force-generating capacity due to the close contact between athletes during the match and during groundwork action (Chaabene et al., 2016; Franchini et al., 2011; Tabben et al., 
2014). Moreover, significant differences were also found between Athletic sprints and other groups regarding $\mathrm{V}_{0}$. Higher velocity and power-generating capacities in the group of Athletic sprints may relate to the specific demands of sprint-running performance. Indeed, sprinters are required to produce high amounts of explosive power (i.e. $\mathrm{P}_{\mathrm{M}}$ ) during push-off phase on the staring block (Harland \& Steele, 1997; Rabita et al., 2015), as well as the ability to produce relatively higher force at high velocity movements (i.e. $\mathrm{V}_{0}$ ) that prevent premature force decline over the acceleration phase and maintain relatively higher force production at maximal speed (Morin et al., 2012). The hypothesized differences observed in the F-V relationship parameters among the groups of elite athletes reflect both the properties and the adaptation mechanisms of the neuromuscular system associated with specifically focused long-term training (Enoka, 1997) and, possibly, the effect of selection (Jenkins, 2012).

Of importance could be the practical implications and limitations of the obtained findings. Taken together, the present findings support the use of the F-V modelling approach obtained from loaded maximal sprints on a cycle ergometer to detect specific training adaptations and talented athletes. The F-V modelling arguably provides a marked advantage over standard methods typically based on single trials when evaluating the effectiveness of various training programs since it allows the separate monitoring of the specific changes in the muscle mechanical capacities, such as high F, V and P output (Jaric, 2015). Such a set of information could be valuable not only in sport but also in other non-clinical (physical education, ergonomy) as well as clinical areas (physical medicine, physical therapy). In addition, using functional movements in testing protocols provides high ecological validity of the assessment of muscle mechanical capacities. Regardless of the generally encouraging results, several potential limitations and directions for future research need to be addressed. Firstly, despite the fact that we applied standard set of loading conditions for testing elite athletes on a leg cycle ergometer (Henry Vandewalle et al., 1987) that allows reliable measurements (García Ramos, Torrejón, Morales Artacho, Pérez Castilla, \& Jaric, 2017), more precise calculation of $P_{M}$ (Pazin et al., 2011) and decreased bias toward $\mathrm{V}_{0}$ or $\mathrm{F}_{0}$ (Pérez-Castilla, Jaric, Feriche, Padial, \& García-Ramos, 2017), it is noted that it was relatively narrow. Therefore, further research should evaluate the sensitivity of the F-V relationship conducted over wider loading conditions. Finally, for the sake of providing a more robust set of data, a similar evaluation on different functional tasks that include both, upper and lower extremity muscle groups, as well as on diverse populations should also be conducted.

Although various standard tests have been found to be sensitive enough to detect differences among various populations, the F-V approach allows assessment of selective changes in the mechanical capacities of lower body muscles associated with chronic practice in a specific sport. While a higher $\mathrm{P}_{\mathrm{M}}$ production has been expected with high-level training, using other $\mathrm{F}-\mathrm{V}$ relation parameters, such as $\mathrm{F}_{0}, \mathrm{~V}_{0}$, and slope, allows the evaluation of specific F-V profiles that enable differentiation of elite athletes from distinct sport settings. Therefore, these results support a high sensitivity of the F-V modelling approach obtained from maximal sprints on a leg cycle ergometer to distinguish among elite athletes with different training histories.

\section{Acknowledgment}

The study was supported by Serbian Institute of Sport and Sports Medicine.

\section{RE F ER E N C E S}

Apostolidis, N., Nassis, G. P., Bolatoglou, T., \& Geladas, N. D. (2004). Physiological and technical characteristics of elite young basketball players. Journal of Sports Medicine and Physical Fitness, 44(2), 157-163.

Chaabene, H., Negra, Y., Bouguezzi, R., Mkaouer, B., Franchini, E., Julio, U., \& Hachana, Y. (2016). Physical and physiological profile of wrestler athletes. Journal of Strength and Conditioning Research, (Epub ahead of print). https://doi.org/10.1519/JSC.0000000000001738

Cohen, J. (1988). Statistical power analysis for the behavioral sciences. Statistical Power Analysis for the Behavioral Sciences. https://doi.org/10.1234/12345678

Cormie, P., McGuigan, M. R., \& Newton, R. U. (2011a). Developing maximal neuromuscular power: Part 1 - Biological basis of maximal power production. Sports Medicine. https://doi.org/10.2165/11537690000000000-00000

Cormie, P., McGuigan, M. R., \& Newton, R. U. (2011b). Developing maximal neuromuscular power: Part 2 training considerations for improving maximal power production. Sports Medicine, 41(2), 125-146. https://doi.org/10.2165/11538500-000000000-00000

Cuk, I., Mirkov, D., Nedeljkovic, A., Kukolj, M., Ugarkovic, D., \& Jaric, S. (2016). Force-velocity property of leg muscles in individuals of different level of physical fitness. Sports Biomechanics, 15(2), 207-219. https://doi.org/10.1080/14763141.2016.1159724

Dotan, R., \& Bar-Or, O. (1983). Load optimization for the wingate anaerobic test. European Journal of Applied Physiology and Occupational Physiology, 51(3), 409-417. https://doi.org/10.1007/BF00429077

Driss, T., Vandewalle, H., Le Chevalier, J.-M., \& Monod, H. (2002). Force-velocity relationship on a cycle ergometer and knee-extensor strength indices. Canadian Journal of Applied Physiology $=$ Revue Canadienne de Physiologie Appliquée, 27(3), 250-62. https://doi.org/10.1139/h02-015

Enoka, R. M. (1997). Neural adaptations with chronic physical activity. Journal of Biomechanics. https://doi. org/10.1016/S0021-9290(96)00170-4 
Evans, J. A., \& Quinney, H. A. (1981). Determination of resistance settings for anaerobic power testing. Canadian Journal of Applied Sport Sciences. Journal Canadien Des Sciences Appliquees Au Sport, 6(2), 53-56.

Feeney, D., Stanhope, S. J., Kaminski, T. W., Machi, A., \& Jaric, S. (2016). Loaded vertical jumping: Forcevelocity relationship, work, and power. Journal of Applied Biomechanics, 32(2), 120-127. https://doi. org/10.1123/jab.2015-0136

Franchini, E., Del Vecchio, F. B., Matsushigue, K. A., \& Artioli, G. G. (2011). Physiological profiles of elite judo athletes. Sports Medicine, 41(2), 147-166. https://doi.org/10.2165/11538580-000000000-00000

Gabbett, T., Georgieff, B., \& Domrow, N. (2007). The use of physiological, anthropometric, and skill data to predict selection in a talent-identified junior volleyball squad. Journal of Sports Sciences, 25(12), 13371344. https://doi.org/10.1080/02640410601188777

García Ramos, A., Torrejón, A., Morales Artacho, A. J., Pérez Castilla, A., \& Jaric, S. (2017). Optimal Resistive Forces For Maximizing The Reliability Of Leg Muscles Capacities Tested On A Cycle Ergometer. Journal of Applied Biomechanics, (Epub ahead of print). https://doi.org/10.1123/jab.2017-0056

Giroux, C., Rabita, G., Chollet, D., \& Guilhem, G. (2016). Optimal balance between force and velocity differs among world-class athletes. Journal of Applied Biomechanics, 32(1), 59-68. https://doi.org/10.1123/ jab.2015-0070

Harland, M. J., \& Steele, J. R. (1997). Biomechanics of the sprint start. Sports Medicine (Auckland, N.Z.), 23(1), 11-20. https://doi.org/10.2165/00007256-199723010-00002

Jaric, S. (2002). Muscle strength testing: Use of normalisation for body size. Sports Medicine, 32(10), 615-631. https://doi.org/321002 [pii]

Jaric, S. (2015). Force-velocity Relationship of Muscles Performing Multi-joint Maximum Performance Tasks. International Journal of Sports Medicine. https://doi.org/10.1055/s-0035-1547283

Jaric, S. (2016). Two-Load Method for Distinguishing Between Muscle Force, Velocity, and Power-Producing Capacities. Sports Medicine, 46(11), 1585-1589. https://doi.org/10.1007/s40279-016-0531-z

Jenkins, S. (2012). Talent Identification and Development in Sport: International Perspectives, Joseph Baker, Steve Cobley and Jörg Schorer (eds.). International Journal of Sports Science and Coaching, 7, 177-180. https://doi.org/10.1260/1747-9541.7.1.177

Jiménez-Reyes, P., Samozino, P., Brughelli, M., \& Morin, J. B. (2017). Effectiveness of an individualized training based on force-velocity profiling during jumping. Frontiers in Physiology, 7(1), 1-13. https://doi. org/10.3389/fphys.2016.00677

Logan, P., Fornasiero, D., Abernethy, P., \& Lynch, K. (2000). Protocols for the Assessment of Isoinertial Strength. In C. J. Gore (Ed.), Physiological Tests for Elite Athletes (pp. 200-222). IL: Human Kinetics.

Massuça, L., \& Fragoso, I. (2013). A multidisciplinary approach of success in team-handball. Apunts Medicina de l'Esport, 48(180), 143-151. https://doi.org/10.1016/j.apunts.2013.06.004

Mendez-Villanueva, A., Bishop, D., \& Hamer, P. (2007). Reproducibility of a 6-s maximal cycling sprint test. Journal of Science and Medicine in Sport, 10(5), 323-326. https://doi.org/10.1016/j.jsams.2006.07.017

Morin, J. B., Bourdin, M., Edouard, P., Peyrot, N., Samozino, P., \& Lacour, J. R. (2012). Mechanical determinants of 100-m sprint running performance. European Journal of Applied Physiology, 112(11), 3921-3930. https://doi.org/10.1007/s00421-012-2379-8

Newton, R., \& Kraemer, W. (1994). Developing explosive muscular power: Implications for a mixed methods training strategy. Strength Condit J, 16(5), 20-31.

Norton, K., Marfell-Jones, M., Whittingham, N., Kerr, D., Carter, L., Saddington, K., \& Gore, C. (2000). Anthropometric Assessment Protocols. In C. Gore (Ed.), Physiological Tests for Elite Athletes (pp. 66-85). IL: Human Kinetics.

Patton, J., Murphy, M., \& Frederick, F. (1985). Maximal Power Outputs During the Wingate Anaerobic Test. International Journal of Sports Medicine, 6(2), 82-85. https://doi.org/10.1055/s-2008-1025818

Pazin, N., Bozic, P., Bobana, B., Nedeljkovic, A., \& Jaric, S. (2011). Optimum loading for maximizing muscle power output: The effect of training history. European Journal of Applied Physiology, 111(9), 2123-2130. https://doi.org/10.1007/s00421-011-1840-4

Pérez-Castilla, A., Jaric, S., Feriche, B., Padial, P., \& García-Ramos, A. (2017). Evaluation of Muscle Mechanical Capacities through the Two-load Method. Journal of Strength and Conditioning Research, (Epub ahead of print). https://doi.org/10.1519/JSC.0000000000001969

Rabita, G., Dorel, S., Slawinski, J., Sàez-de-Villarreal, E., Couturier, A., Samozino, P., \& Morin, J. B. (2015). Sprint mechanics in world-class athletes: A new insight into the limits of human locomotion. Scandinavian Journal of Medicine and Science in Sports, 25(5), 583-594. https://doi.org/10.1111/sms.12389

Ravier, G., Grappe, F., \& Rouillon, J. D. (2004). Application of force-velocity cycle ergometer test and vertical jump tests in the functional assessment of karate competitor. Journal of Sports Medicine and Physical Fitness, 44(4), 349-355.

Reilly, T., Williams, A. M., Nevill, A., \& Franks, A. (2000). A multidisciplinary approach to talent identification in soccer. Journal of Sports Sciences, 18(9), 695-702. https://doi.org/10.1080/02640410050120078

Samozino, P., Edouard, P., Sangnier, S., Brughelli, M., Gimenez, P., \& Morin, J. B. (2014). Force-velocity profile: Imbalance determination and effect on lower limb ballistic performance. International Journal of Sports Medicine, 35(6), 505-510. https://doi.org/10.1055/s-0033-1354382

Samozino, P., Rejc, E., Di Prampero, P. E., Belli, A., \& Morin, J. B. (2012). Optimal force-velocity profile in 
ballistic movements-Altius: Citius or Fortius? Medicine and Science in Sports and Exercise, 44(2), 313322. https://doi.org/10.1249/MSS.0b013e31822d757a

Tabben, M., Chaouachi, A., Mahfoudhi, M., Aloui, A., Habacha, H., Tourny, C., \& Franchini, E. (2014). Physical and physiological characteristics of high-level combat sport athletes. Journal of Combat Sports and Martial Arts, 5(1), 1-5. https://doi.org/10.5604/20815735.1127445

Vandewalle, H., Péerès, G., \& Monod, H. (1987). Standard Anaerobic Exercise Tests. Sports Medicine: An International Journal of Applied Medicine and Science in Sport and Exercise. https://doi. org/10.2165/00007256-198704040-00004

Vandewalle, H., Peres, G., Heller, J., Panel, J., \& Monod, H. (1987). Force-velocity relationship and maximal power on a cycle ergometer - Correlation with the height of a vertical jump. European Journal of Applied Physiology and Occupational Physiology, 56(6), 650-656. https://doi.org/10.1007/BF00424805

Westing, S. H., Seger, J. Y., \& Thorstensson, A. (1990). Effects of electrical stimulation on eccentric and concentric torque-velocity relationships during knee extension in man. Acta Physiologica Scandinavica, 140(140), 17-22. https://doi.org/10.1111/j.1748-1716.1990.tb08971.x

Wilson, G. J., Newton, R. U., Murphy, A. J., \& Humphries, B. J. (1993). The optimal training load for the development of dynamic athletic performance. Medicine \& Science in Sports \& Exercise, 25(11), 12791286. https://doi.org/10.1249/00005768-199311000-00013 